\title{
Adhesion to Vitronectin and Collagen I Promotes Osteogenic Differentiation of Human Mesenchymal Stem Cells
}

\author{
Roman M. Salasznyk, ${ }^{1}$ William A. Williams, ${ }^{1}$ Adele Boskey, ${ }^{2}$ Anna Batorsky, ${ }^{1}$ and George E. Plopper ${ }^{1 *}$ \\ ${ }^{1}$ Department of Biology, Rensselaer Polytechnic Institute, Troy, NY 12180-3596, USA \\ ${ }^{2}$ Hospital for Special Surgery, New York City, NY 10021, USA
}

Received 3 June 2003; revised 9 July 2003; accepted 9 July 2003

\begin{abstract}
The mechanisms controlling human mesenchymal stem cells (hMSC) differentiation are not entirely understood. We hypothesized that the contact with extracellular matrix (ECM) proteins normally found in bone marrow would promote osteogenic differentiation of hMSC in vitro. To test this hypothesis, we cultured hMSC on purified ECM proteins in the presence or absence of soluble osteogenic supplements, and assayed for the presence of well-established differentiation markers (production of mineralized matrix, osteopontin, osteocalcin, collagen I, and alkaline phosphatase expression) over a 16-day time course. We found that hMSC adhere to ECM proteins with varying affinity (fibronectin $>$ collagen I $\geq$ collagen IV $\geq$ vitronectin $>$ laminin-1) and through distinct integrin receptors. Importantly, the greatest osteogenic differentiation occurred in cells plated on vitronectin and collagen I and almost no differentiation took place on fibronectin or uncoated plates. We conclude that the contact with vitronectin and collagen I promotes the osteogenic differentiation of hMSC, and that ECM contact alone may be sufficient to induce differentiation in these cells.
\end{abstract}

\section{INTRODUCTION}

Human mesenchymal stem cells (hMSC) are a population of multipotent cells located within the bone marrow and are characterized by their ability to differentiate into at least three phenotypes when cultured in vitro $[1,2]$. These cells are related to, but distinct from, stromal stem cells, and are carefully selected for uniform expression of approximately 50 surface antigens (with $98 \%$ homogeneity at passage 2), including at least three different stem cell surface marker proteins $[1,2]$. Once isolated and purified from the marrow stroma, uncommitted hMSC retain the capacity to self-renew and differentiate along multiple pathways resulting in the generation and maintenance of an assortment of tissues. This in turn raises the possibility of utilizing these cells to repair or replace damaged tissues. A more complete understanding of the molecular mechanisms driving the differentiation of these cells should significantly facilitate the adoption of these cells in clinical applications.

What drives the differentiation of hMSC is not entirely known. The development of these mesenchymal progenitors along an osteogenic [3], chondrogenic [4], and adipogenic [1] linage occurs primarily under the influence of chemical stimuli (eg, dexamethazone, transforming growth factor $\beta 3$, insulin), which is accompanied by profound changes in morphology, proliferation, gene expression, and molecular signaling events. However, the underlying mechanisms governing mesenchymal stem cell differentiation in vitro and in vivo are not yet completely understood.

Cellular differentiation is induced by cues in the environment immediately surrounding cells. Many of the soluble cues known to influence hMSC differentiation have been identified. For example, when hMSC are cultured in the presence of ascorbic acid-2-phosphate (AsAP), $\beta$ glycerophosphate $(\beta \mathrm{GP})$, and the synthetic glucocorticoid dexamethasone (Dex), they adopt an osteoblastic phenotype and secrete and organize an extracellular matrix (ECM) where calcium phosphate is deposited as hydroxyapatite crystals [5]. Induction by this osteogenic supplement (OS) medium triggers a series of molecular events including activation of signal transduction pathways and expression of osteogenic marker genes including collagen I (COL I), osteocalcin, osteopontin, and alkaline phosphatase $[1,6,7,8]$. While these media supplements clearly induce osteoblast differentiation under some conditions, they may suppress bone growth in vivo $[9,10]$, which may limit their usefulness for repairing bone in situ. Response to these agents is biphasic, concentration-dependent, and varies with the length of exposure $[6,11]$. The result is a somewhat heterogeneous population of cells with mixed differentiation potential [12]. There is a clear need to better understand the molecular mechanisms that control osteogenesis in these cells.

Insoluble cues that affect cellular differentiation arise largely from cellular binding to ECM proteins. While considerable attention has been paid to the role of OS 
TABle 1. Primers used for RT-PCR.

\begin{tabular}{llc}
\hline GENE/NCBI designation & Primer sequences & Reference \\
\hline $\begin{array}{l}\text { GAPDH (glyceraldehyde-3- } \\
\text { phosphate dehydrogenase) }\end{array}$ & $\begin{array}{l}\text { Forward 5'-ATGGAAATCCCATCACCATCT-3' } \\
\text { Reverse 5' -GGTTGAGCACAGGGTACTTTATT-3' }\end{array}$ & (Doi et al [7]) \\
\hline ALPL (alkaline phosphatase) & $\begin{array}{l}\text { Forward 5'-GACCCTTGACCCCCACAAT-3' } \\
\text { Reverse 5' -GCTCGTACTGCATGTCCCCT-3' }\end{array}$ & (Frank et al [23]) \\
\hline COL I (collagen type I) & $\begin{array}{l}\text { Forward 5'-CAGCCGCTTCACCTACAGC-3' } \\
\text { Reverse 5' -TTTTGTATTCAATCACTGTCTTGCC-3' }\end{array}$ & (Frank et al [23]) \\
\hline SPP1 (osteopontin) & $\begin{array}{l}\text { Forward 5'-CTCAGGCCAGTTGCAGCC-3' } \\
\text { Reverse 5' -CAAAAGCAAATCACTGCAATTCTC-3' }\end{array}$ & (Frank et al [23]) \\
\hline $\begin{array}{l}\text { BGLAP (bone gamma-carboxyglutamate } \\
\text { (gla) protein/osteocalcin) }\end{array}$ & $\begin{array}{l}\text { Forward 5'-GAAGCCCAGCGGTGCA-3' } \\
\text { Reverse 5' -CACTACCTCGCTGCCCTCC-3' }\end{array}$ & (Frank et al [23]) \\
\hline
\end{tabular}

medium in hMSC differentiation, comparatively little is known about the effect of ECM binding hMSC behavior. However, it is well known that ECM proteins play a major role in bone development. For example, during endochondral bone development, collagen II expression peaks during the chondrogenesis period while COL I deposition is maximal during the ossification phase [13]. Furthermore, it has been known for decades that single-point mutations in COL I yield a lethal form of osteogenesis imperfecta (eg, Pace et al [14]). In other studies, genetic knockouts of collagen II in mice result in severe developmental abnormalities and skeletal defects, especially in cartilaginous elements [15]. In vitro, bone marrow stromal cells undergo osteogenic differentiation when cultured on COL I matrix, and this requires interaction with the COL-I-binding integrin $\alpha 2 \beta 1[16,17]$. The mechanisms linking ECM binding to osteogenic differentiation, especially in hMSC, are largely unknown.

ECM proteins typically affect cell behavior by binding to specific integrin cell surface receptors, which activate intracellular signaling pathways and control gene expression, cytoskeletal organization, and cell morphology. Over 20 different integrin receptors have been identified, which bind to well over 20 different ECM proteins and stimulate at least six different classes of intracellular signaling molecules: protein tyrosine kinases, serine/threonine kinases, lipid kinases, lipid phosphatases, protein phosphatases, and intracellular ion fluxes [18]. The contribution of integrin-associated pathways to osteogenic differentiation of hMSC is largely unknown. Recent evidence implicates ERK 1/2, an integrin- and growth factor-associated signaling protein, as playing a critical role in osteogenic differentiation of hMSC in vitro [1]. The role of ECM proteins in controlling ERK 1/2 activity and other signaling molecules during osteogenesis has not been investigated.

How hMSC translate adhesion to ECM into cell differentiation is entirely unknown. We hypothesized that adhesion to ECM proteins, in the absence of soluble osteogenic stimulants, would enhance the osteogenic differentiation of hMSC. To test this hypothesis, we cultured hMSC on purified ECM proteins for 16 days in the presence or absence of soluble osteogenic supplements and assessed the differentiation of these cells by a variety of markers. Our results demonstrate that contact with COL I or vitronectin alone may be sufficient to induce differentiation in these cells.

\section{MATERIALS AND METHODS}

\section{Materials}

Purified bovine COL I, human plasma vitronectin, and human plasma fibronectin proteins were purchased from Chemicon International (Temecula, Calif). Purified mouse collagen IV (COL IV) and mouse laminin-1 proteins were purchased from Collaborative Research Inc (Bedford, Mass). The $\alpha 1-6$ and $\beta 1-4$ integrin functionblocking antibodies (Alpha integrin blocking and IHC kit, catalog \# ECM 430; Beta integrin screening kit, catalog \# ECM 440) and anti-human, rabbit IgG antibodies against osteopontin (catalog \# AB1870) were obtained from Chemicon International (Temecula, Calif). Antihuman, mouse IgG antibody against tubulin was obtained from Sigma (St Louis, Mo) (catalog \# T5168). Phalloidin conjugated to rhodamine (TRITC) was purchased from Molecular Probes (Eugene, Ore). Reverse transcriptase-polymerase chain reaction RT-PCR primers listed in Table 1 were purchased from IDT Technologies (Coralville, Iawa).

\section{Culture of hMSC and human osteoblasts}

Cryopreserved hMSC were purchased from Cambrex Inc (Walkersville, Md) and grown as directed. Briefly, cells were plated at $5 \times 10^{3}$ cells $/ \mathrm{cm}^{2}$ in a T75 flask $\left(75 \mathrm{~cm}^{2}\right)$ for continuous passaging in control medium (DMEM supplemented with $10 \%$ fetal calf serum, $1 \%$ L-glutamine [29.2 mg/mL], penicillin $\mathrm{G}[10,000$ units $/ \mathrm{mL}]$ and streptomycin sulfate $[10,000 \mu \mathrm{g} / \mathrm{mL}])$. The OS medium was control medium (labeled "DMEM" in Figures) supplemented with $0.1 \mu \mathrm{M}$ Dex, $0.05 \mathrm{mM}$ AsAP, and $10 \mathrm{mM} \beta G P$ (Sigma Chemical Co, St Louis, Mo).

For in vitro osteogenic assays, hMSC were passaged three times before they were inducted and plated at densities of $3.1 \times 10^{3}$ cells $/ \mathrm{cm}^{2}$ in $0.2 \mathrm{~mL} / \mathrm{cm}^{2}$ of control medium in either $60 \mathrm{~mm}$ Falcon dishes $\left(28.0 \mathrm{~cm}^{2}\right)$, 
24-well plates $\left(2.00 \mathrm{~cm}^{2}\right)$, or 96 -well plates $\left(0.32 \mathrm{~cm}^{2}\right)$ according to the specification of the assays described below. Cultures were incubated at $37^{\circ} \mathrm{C}$ in a humidified atmosphere containing $95 \%$ air and $5 \% \mathrm{CO}_{2}$. The following day (day 0 ), we replaced the culture medium with OS or fresh control medium. Media changes were performed twice weekly at volumes described above. At days $4,8,12$, and 16 , cultures were assayed as described below.

Isolated human osteoblasts (purchased from Cambrex) were grown according to manufacturers' instructions in a growth medium (basal medium supplemented with $10 \%$ fetal calf serum, ascorbic acid, gentamycin, and amphotericin B). Following one week in culture, cells were replated in growth media supplemented with $200 \mathrm{mM}$ hydrocortisone-21-hemisuccinate and $10 \mathrm{mM}$ $\beta$ GP. Eight days later, cells were harvested and RNA was isolated for RT-PCR as described below.

\section{Adhesion assays}

Cell adhesion assays were performed as previously described using Sarstedt 96-well suspension cell culture plates [19]. Tissue culture plates were coated with purified ECM proteins (collagen I+IV, fibronectin, laminin-1, and vitronectin) at a concentration of $20 \mu \mathrm{g} / \mathrm{mL}$ for 1 hour at room temperature. Wells were washed twice with PBS and incubated with nd-blotto (5\% non-diary creamer in PBS $+0.2 \%$ Tween 20 ) for 30 minutes prior to the assay. hMSC were seeded as described above on either plastic substrata or ECM proteins, and allowed to attach for 30 minutes at $37^{\circ} \mathrm{C}$. Cells were subsequently fixed with $3 \%$ paraformaldehyde, washed twice in PBS, and incubated in crystal violet dye for 15 minutes. Wells were washed thoroughly with water and the violet dye was extracted with $10 \%$ SDS solution. Absorbance was measured using a TECAN SPECTRAFluor spectrophotometer at $595 \mathrm{~nm}$ and relative adhesion was compared to cells attached to nd-blotto.

Integrin-blocking adhesion assays were performed according to the procedure above, but the cells were incubated with a functional integrin-blocking antibody for 30 minutes at $37^{\circ} \mathrm{C}$, with vortexing every 5 minutes, prior to plating.

\section{Immunohistochemistry}

Tubulin, F-actin, and osteopontin were localized in hMSC cultured on glass coverslips as previously described [19]. Briefly, primary antibodies or phalloidin (diluted 1:200 in PBS) were added for 1 hour to cells fixed in $3 \%$ paraformaldehyde and blocked with $\mathrm{PBS} / 1 \% \mathrm{BSA}$. Secondary antibodies conjugated to fluorescein or rhodamine (Jackson Immuno Research, Inc, West Grove, Pa) were added for 1 hour, then coverslips were mounted using Prolong antifade medium (Molecular Probes, Eugene, Ore). Cells were visualized with a Nikon TE2000-S inverted fluorescence/phase contrast microscope equipped with a digital SPOT camera.

\section{Western blot analysis}

Proteins were separated by $8 \%$ SDS-PAGE and transferred to Immobilon-P transfer membranes from Millipore (Bedford, Mass) and processed for immunoblotting as previously described [20]. Processed membranes incubated with primary antibody overnight were then incubated with secondary goat anti-rabbit IgG-HRP from Pierce Biotechnology (Rockford, Ill) and exposed to UnBlot Chemiluminescent reagent from Pierce Biotechnology. Images were detected with a ChemiImager $4400 \mathrm{Gel}$ imaging system (Alpha Innotech, San Leandro, Calif).

\section{Histological staining of alkaline phosphatase activity and calcium precipitation}

At days 4, 8, 12, and 16, cell cultures were assayed as described below. For the detection of alkaline phosphatase activity, a solution of naphthol AS-MX phosphate and fast blue RR dissolved in distilled (d) $\mathrm{H}_{2} \mathrm{O}$ was poured into wells of a 24-well plate according to the manufacturer's instructions contained in Sigma Kit \#85. Cellular specimens were scored according to the quantity and intensity of the precipitated dye. For the detection of a calciumphosphate-containing matrix, cell layers were stained by the von Kossa method [21]. Specimens were fixed in $3 \%$ paraformaldhyde for 30 minutes. and subsequently washed twice in $\mathrm{dH}_{2} \mathrm{O}$ and incubated in the dark with $5 \%$ silver nitrate solution for 10 minutes. Afterwards, the cell layers were washed thoroughly with $\mathrm{dH}_{2} \mathrm{O}$ and then exposed to bright sunlight for 1 hour. Cellular specimens were scored according to the quantity and size of precipitated granules.

\section{Calcium assay}

Specimens were washed twice with PBS and extracted off a well of a 24 -well plate in $0.5 \mathrm{~N} \mathrm{HCl}$. Accumulated calcium was removed from the cellular component by shaking for 5 hours at $4^{\circ} \mathrm{C}$, followed by centrifugation at 2,000 g for 10 minutes. The consequent supernatant was utilized for calcium determination according to the manufacturer's instructions contained in Sigma Kit \#587. Total calcium was calculated from standard solutions prepared in parallel and expressed as ng/well after absorbance at $575 \mathrm{~nm}$ was measured.

\section{Mineralized bone: Fourier transform infrared (FTIR) analysis}

The presence of apatite in cell matrix was detected by FTIR of ground powders. Cell layers, collected in $50 \mathrm{mM}$ ammonium bicarbonate ( $\mathrm{pH} 8.0$ ), were lyophilized and analyzed as potassium bromide $(\mathrm{KBr})$ pellets on a BioRad FTS 40-A spectrometer (Bio-Rad, Cambridge, Mass, USA). The data was collected under nitrogen purge, and the spectral baseline corrected and analyzed using GRAMS/386 software (Galactic Industries, Salem, NH, USA) as previously described [22]. The mineral content is calculated based on the spectrally derived mineralto-matrix ratio (the integrated areas of the phosphate 


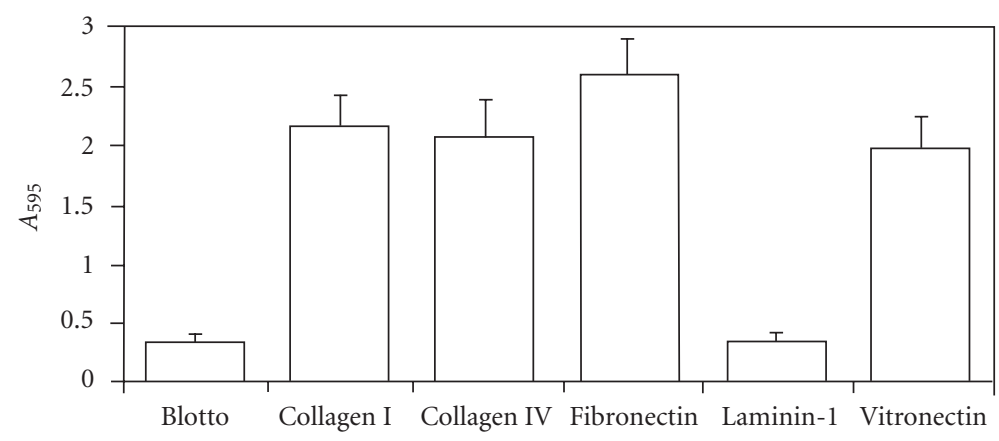

FIGURE 1. Static 30-minute assays of hMSC adhesion to purified extracellular matrix proteins. Adherent cells were stained with crystal violet, then solubilized in SDS and absorbance determined at $595 \mathrm{~nm}$. Values represent mean \pm standard deviation $(n=5)$.

absorbance $\left(900-1200 \mathrm{~cm}^{-1}\right)$ and protein amide I band $\left.\left(1585-1720 \mathrm{~cm}^{-1}\right)\right)$.

\section{Reverse transcriptase-polymerase chain reaction}

RNA was isolated from $10 \times 10^{8} \mathrm{hMSC}$ cultured for eight days on tissue culture plastic in the presence or absence of OS, or on COL I, or VN in control media. As a positive control for differentiation, RNA was isolated from $10 \times 10^{8}$ human osteoblasts cultured for eight days. Total RNA was isolated using the RNeasy mini kit (Qiagen, Valencia, Calif). RT-PCR was performed with the OneStep RT-PCR Kit (Qiagen) and a 96-well thermal cycler (MJ Research, Waltham, Mass) using the primers listed in Table 1, which were obtained from [7, 23]. One microgram of template RNA was used per reaction. The reverse transcription step ran for 30 minutes at $50^{\circ} \mathrm{C}$, followed by PCR activation for 15 minutes at $95^{\circ} \mathrm{C}$. Thirty amplification cycles were run, consisting of one minute denaturation at $94^{\circ} \mathrm{C}$, one minute of annealing at $58^{\circ} \mathrm{C}$, and one minute of extension at $72^{\circ} \mathrm{C}$. Final extension was allowed to run 10 minutes at $72^{\circ} \mathrm{C}$. Reaction products were separated by gel electrophoresis using a $2 \%$ agarose gel. Bands were visualized by UV illumination of ethidium-bromide-stained gels and captured using a Chemilmager $4400 \mathrm{Gel}$ imaging system (Alpha Innotech).

\section{RESULTS}

\section{HMSC adhesion to purified ECM proteins}

To assess the contribution of extracellular matrix proteins to the osteogenic differentiation of hMSC, we first examined the ability of these cells to adhere to ECM proteins found in bone marrow. In 30-minute adhesion assays, we found that fibronectin, COL I, COL IV, and vitronectin supported approximately 6 - to 8 -fold greater static adhesion compared to the negative control (ndblotto) or laminin-1 (Figure 1). Approximately $75 \%-80 \%$ of cells adhered to fibronectin, as assessed by visual inspection before and after washing steps. Adhesion to the indicated ECM proteins was blocked more than $90 \%$ by antibodies against the following integrins: COL I, $\alpha 1$ and $\beta 1$; COL IV, $\alpha 1$ and $\beta 1$; FN, $\alpha 5$ and $\beta 1$; and $\mathrm{VN}, \alpha \mathrm{V}$ and $\beta 3$ (not shown).

\section{HMSC morphology and arrangement of microtubules and actin filaments}

We observed culture medium-dependent differences in overall cellular morphology in cells plated on purified ECM proteins for up to 16 days. For example, cells plated on vitronectin $(\mathrm{VN})$ in control medium had a more linear arrangement of the microtubule cytoskeleton than those plated on OS medium (Figures 2a and 2b). Cells plated on COL I also showed these morphological differences, and displayed a rearrangement of actin bundles when cultured in OS medium (Figures $2 \mathrm{c}$ and 2d). We also observed extensive infoldings of the plasma membrane, resulting in the appearance of an array of filopodia- and lamellopodia-like structures in OS-treated cells. Yet these projections lacked organized microtubules (Figure 2b), actin filaments (Figure 2d), and focal adhesions (not shown). This effect was seen in cells plated on all substrates tested, including glass, and appeared to be an effect of the OS-media rather than the substrates. No statistical difference in overall surface area was found between control or OS-treated cells on any substrate (not shown).

\section{Binding to vitronectin and $\mathrm{COL}$ I stimulates osteogenic differentiation of hMSC}

We assessed osteogenic differentiation of our cultures by a number of criteria. First, we determined the mineral to matrix ratio of the secreted ECM, a hallmark of ossification (Table 2). We found that culturing hMSC on purified VN or COL I, even in the absence of OS stimulants, is sufficient to induce mineralization, and hence osteogenic differentiation, after only 16 days. When compared to control cultures of fully differentiated osteoblasts (Figure 3a), the Fourier transform infrared (FTIR) spectroscopic assessment of mineral content from hMSC cultured on COL I (Figure 3c) and VN (Figure 3d) agree well, while the spectrum from cells plated on tissue culture plastic in control medium lacks a significant phosphate 


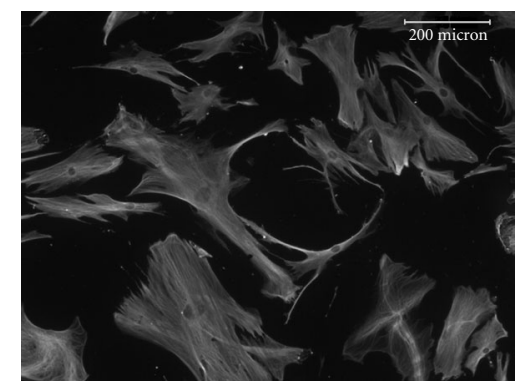

(a)

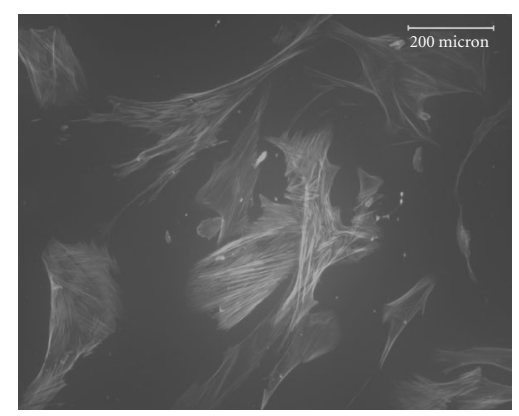

(c)

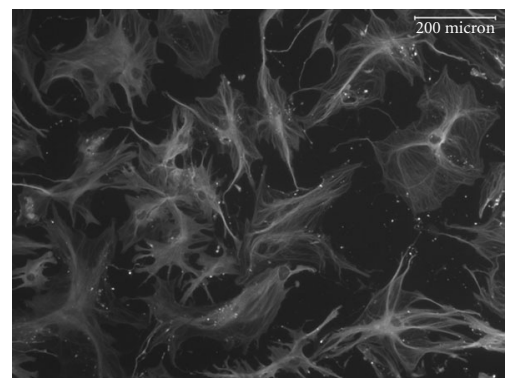

(b)

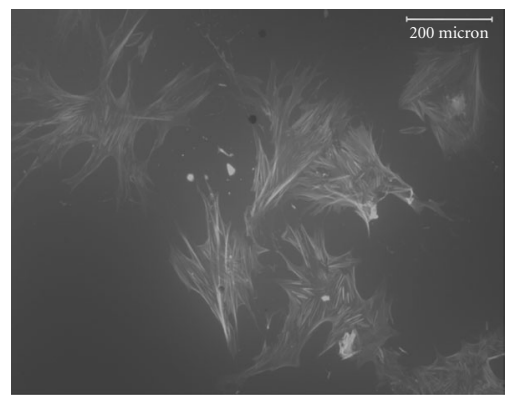

(d)

FIGURE 2. Rearrangement of microtubules and actin filaments in OS-treated cells. HMSC were plated on VN for 16 days in control (a) or OS-containing medium (b), or on COL I for 16 days in control medium (c) or in OS medium (d) then fixed and stained for tubulin using primary antitubulin antibody (a) and (b) or for F-actin using TRITC-phalloidin (c) and (d). Bar = 200 micrometers.

TABLE 2. Osteogenic differentiation of hMSC plated on indicated substrates for 16 days, as assessed by indicated measurements of mineral to matrix ratio. By comparison, fully differentiated osteoblasts cultured on tissue culture plastic for 21 days have a ratio of approximately 5.4 .

\begin{tabular}{l|c}
\hline HMSC plated on substrate/medium & Mineral to matrix ratio \\
\hline Tissue culture plastic/DMEM & 1.662 \\
Tissue culture plastic/OS & 1.515 \\
Collagen I/DMEM & 1.763 \\
Collagen I/OS & 2.372 \\
Vitronectin/DMEM & 2.767 \\
Vitronectin/OS & 2.761 \\
\hline
\end{tabular}

peak (Figure 3b). Mineral to matrix ratios of differentiated osteoblasts are approximately 5.4 after 21 days in culture (A. Boskey, unpublished observation) suggesting that $\mathrm{VN}$ and COL I induce proper mineralization of hMSC matrix in the absence of OS media, and that this mineralization is approximately half that of mature osteoblasts after only 16 days.

Our mineralization data strongly support our supposition that ECM stimuli alone play an important role in inducing osteogenesis of hMSC. Over the same time course, we found that COL I and $\mathrm{VN}$ also supported calcification of the matrix, as assessed by measurements of total calcium [24] (Figure 4) and by the von Kossa silver nitrate staining method for secreted calcium salt [21] (Figure 5).

Immunohistochemical localization (Figure 6a and 6b) and western blotting (Figure 6b) of the early osteogenic marker protein osteopontin revealed distinct accumulation of this protein in cells plated on COL I and VN; this accumulation was not seen in cells plated on tissue culture plastic (Figure 6b, lane 1).

Though not specific for osteoblasts, expression of alkaline phosphatase (AP) is a well-established marker for differentiation along the osteogenic pathway $[6,25]$. Plating cells in control medium on COL I and VN for 16 days increased AP activity relative to control cells plated on glass; addition of traditional osteogenic supplements exacerbated these effects (Figure 7). In the control group, approximately $40 \%$ of cells stained positive for AP activity by day 16 , while at least $80 \%$ of cells in the OS group were positive by day 16 (data not shown).

Collectively, these data suggest that plating cells on COL I or $\mathrm{VN}$ induces osteogeneic differentiation of hMSC. To confirm our findings, we performed RT-PCR analysis for three osteogenic marker genes: COL I, osteopontin, and alkaline phosphatase. Our results (Figure 8) demonstrate that COL I and VN support expression of all 


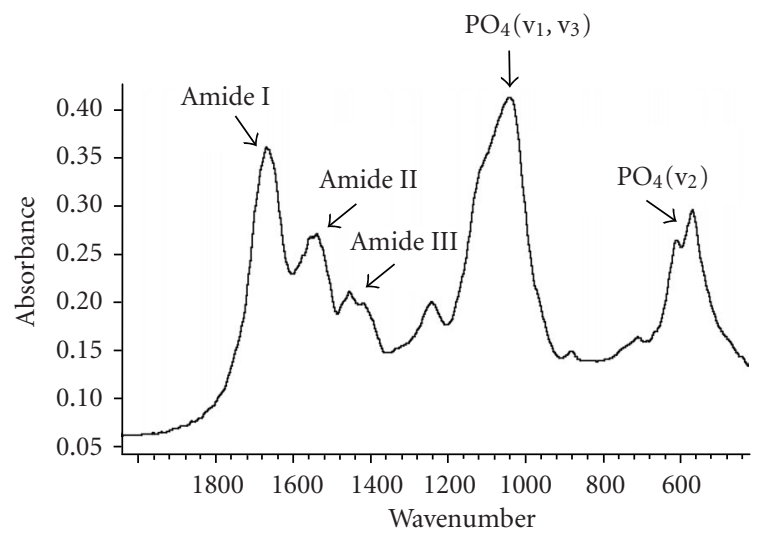

(a)

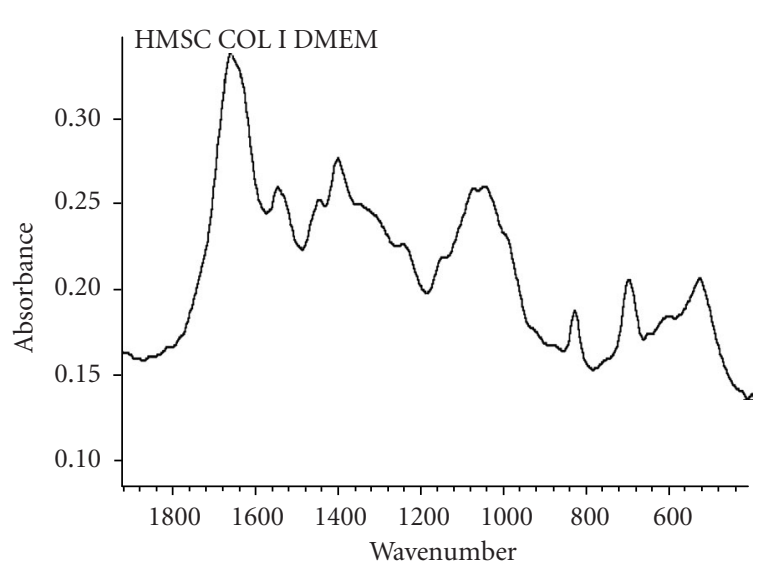

(c)

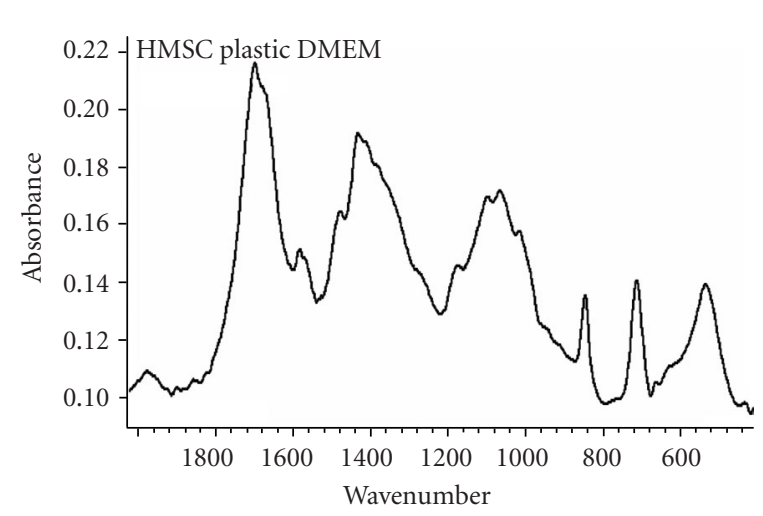

(b)

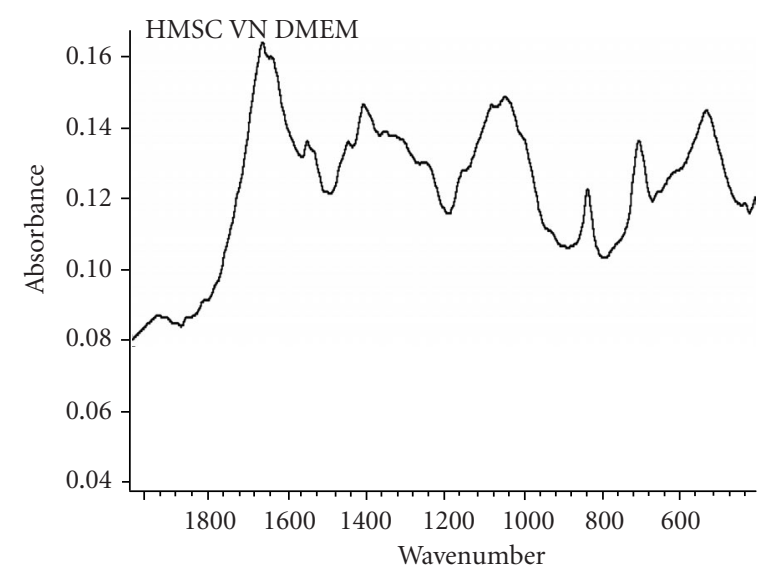

(d)

FIGURE 3. Spectroscopic analysis of apatite in hMSC cultures. For comparison, the profile for matrix secreted by mature osteoblasts cultured for 21 days is shown in (a). The hMSC were plated under the indicated conditions (b), (c), and (d) for 16 days, then the matrix was collected and analyzed by FTIR.

three genes in hMSC after eight days in culture. In particular, osteopontin expression was highest in cells plated on VN (lane 4). In addition, COL I expression was greatest in cells plated on COL I (lane 3). Fully differentiated osteoblasts (lane 5) expressed relatively high amounts of all three genes tested, consistent with their differentiated phenotype.

\section{DISCUSSION}

Our data demonstrate a strong and rapid (30 minutes) adhesive interaction between hMSC and four of the five major ECM proteins found in bone marrow (COL I, VN, COL IV, fibronectin). The lack of rapid adhesion to laminin-1 may be explained by the reported absence of the laminin receptor $\alpha 3 \beta 1$ integrin in preosteoblasts [33]. The hMSC do attach specifically to laminin-1, but only after two hours (not shown). Gronthos et al [33] have demonstrated that stromal stem cells proliferate on all ECM proteins we tested, including laminin-1, using a 14-day colony-forming assay. We have observed that plating hMSC on purified ECM proteins, including laminin1 , supports growth for at least 16 days. It is therefore likely that all ECM proteins we tested play a role in some stage of osteogenesis.

Some differences between our results and those for stromal stem cells and osteoblasts are noteworthy. For example, we find little role for fibronectin in stimulating osteogenic differentiation, beyond activation of AP activity (not shown). However, Damsky and colleagues $[26,27$, 28] have demonstrated a role for fibronectin during calvarial osteoblast differentiation in vitro. Yu et al [29] identified increased fibronectin expression during the chondrogenesis phase of bone formation in vivo. Fibronectin expression varies significantly during the time course of mesenchymal condensation, chondrogenesis, and bone formation, and is expressed as splice variants. It is therefore possible that the window of differentiation we have observed with hMSC represents a stage largely independent of fibronectin's effects. Alternatively, the requirement 


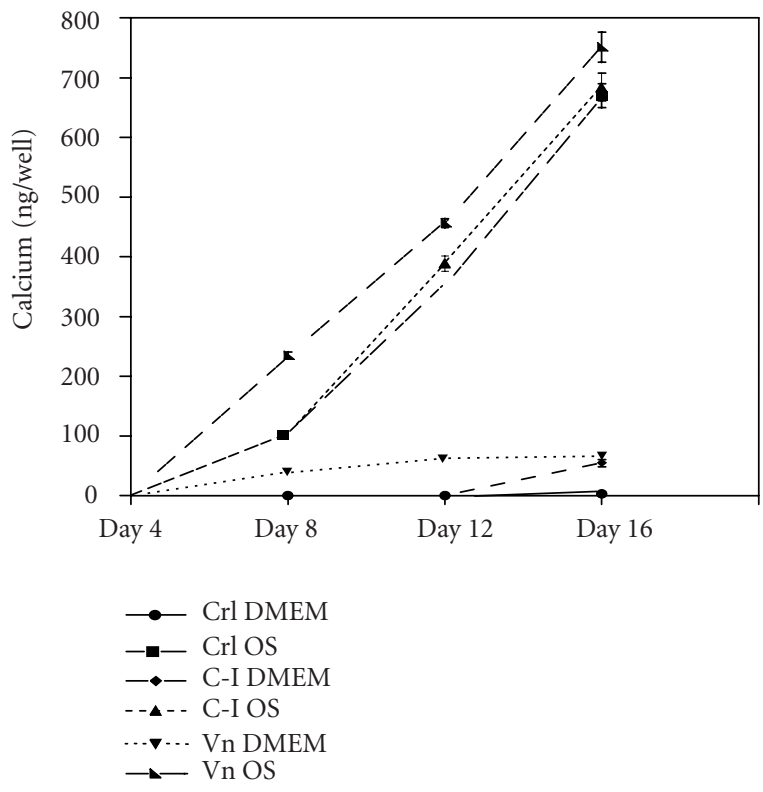

Figure 4. The hMSC produce a calcified matrix when plated on COL I and VN. (a) Cells were plated on tissue culture plastic (CTL), COL I (C-I), or vitronectin (VN) in control medium (DMEM) or media supplemented with osteogeneic supplements (OS) for the indicated time, then total calcium in each well was determined using a colorimetric assay.

TABLE 3. Values of total calcium in each well for all samples at day 16. The values were determined using a colorimetric assay.

\begin{tabular}{ll}
\hline HMSC plated on substrate/medium & Total calcium (ng/well) \\
\hline Tissue culture plastic/DMEM & $3.204 \pm 0.73$ \\
Tissue culture plastic/OS & $669.73 \pm 20.06$ \\
Vetronectin/DMEM & $65.79 \pm 4.56$ \\
Vetronectin/OS & $751.21 \pm 25.32$ \\
Collagen I/DMEM & $54.43 \pm 6.14$ \\
Collagen I/OS & $684.22 \pm 23.38$ \\
Collagen IV/DMEM & $25.94 \pm 2.45$ \\
Collagen IV/OS & $645.92 \pm 21.87$ \\
Fibronectin/OS & $663.06 \pm 18.35$ \\
Laminin-1/OS & $613.47 \pm 15.21$ \\
\hline
\end{tabular}

for fibronectin may be tissue specific, such that calvarial osteoblasts require fibronectin while bone-marrowderived stem cells do not.

COL I has been reported to induce calcification of stromal cell matrix after 3 weeks [16] while we find that both $\mathrm{VN}$ and COL I support the greatest increase in mineral to matrix ratio, calcium deposition, and osteopontin/osteocalcin secretion. These increases are observed in both control media and OS-containing media; as expected, the effects are significantly increased in OS media (Figure 4 and Table 3), suggesting that contact with ECM alone induces differentiation but also enhances the sensitivity to OS media. The differentiation we observe in hMSC occurs within 16 days rather than 21 days, as re- ported for stromal cells. These results may be explained by the fact that stromal cells and hMSC, while being clearly similar, are isolated using different stem cell markers $[2,30,31,32]$. From the combined results from both cell types we conclude that multiple ECM proteins may provide a suitable substrate for the attachment of hMSC to the underlying matrix, but that signaling through the COL I receptor $\alpha 1 \beta 1$ integrin and the $\mathrm{VN}$ receptor $\alpha \mathrm{V} \beta 3$ integrin plays the most significant role in promoting osteogenesis. Incubation of preosteoblasts with a functionblocking antibody against the integrin $\alpha 1$ subunit inhibits matrix mineralization [33], further implicating $\alpha 1 \beta 1$ as a significant player in this process.

The hMSC have been reported to undergo a morphological transition from an elongated, spindle shape to a cuboidal shape during OS-induced differentiation [3]. Our results support this observation, and based on our examination of the microtubule and actin cytoskeleton, we conclude that OS components induce dramatic remodeling of the entire cytoskeletal network. A drop in parallel microtubule bundles and actin stress fibers in OStreated cells, accompanied by extensive infolding of the plasma membrane, suggests that cells are transitioning from a tightly adhesive to a more loosely adherent phenotype. The total surface area in control and OS-treated cells did not differ significantly (not shown), so cell spreading appears to be not directly affected. But the absence of focal complexes or focal adhesions in these OS-induced projections suggests that they are not filopodia or lamellopodia, and that OS-treated cells are not motile. Interestingly, however, we found that cells plated on $\mathrm{VN}$ or COL I in control medium lacked these projections and yet underwent the initial stages of ossification, suggesting that hMSC may retain some migration ability in the early phases of differentiation. This raises an additional concern regarding the use of OS components for in vivo application of hMSC. In particular, this could have important implications for tissue engineering of bone, where proper organization of osteoblasts in implants is essential, and may require stem cell migration following polymer seeding $[34,35]$. Our results suggest that OS components may not be necessary for proper differentiation of hMSC in engineered bone.

Our differentiation data suggest that adhesion to purified $\mathrm{VN}$, in addition to COL I, is a potent inducer of hMSC osteogenesis. However, it is certain that osteogenesis requires contact with COL I. This in turn suggests that $\mathrm{VN}$ and COL I may act cooperatively during differentiation. While the $\alpha \mathrm{v} \beta 3$ integrin binds to RGD sites in COL $\mathrm{I}$, these sites are cryptic and are only exposed after proteolytic processing of native COL I [34]. The $\alpha \mathrm{v} \beta 3$ integrin also binds to the RGD sequence in osteopontin $[9,36]$, and thus may participate in later stages of differentiation, following osteopontin expression. We find expression of COL I in VN cultures at days 12 and 16 (not shown). The presence of both VN and COL I in the "VN" samples, especially at later time points, may engage multiple 


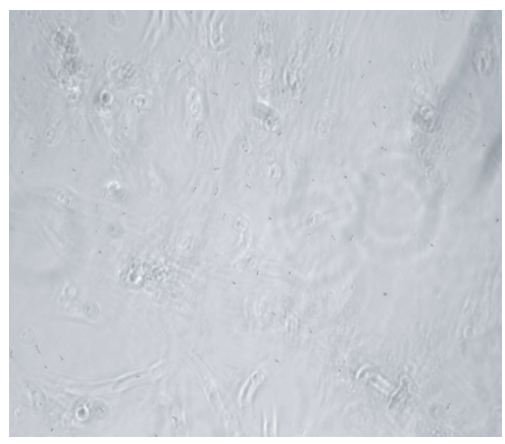

(a)

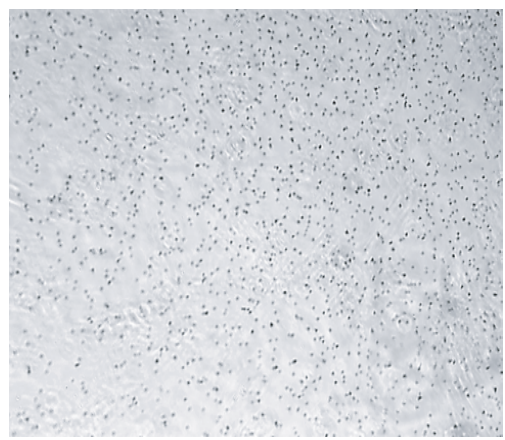

(d)

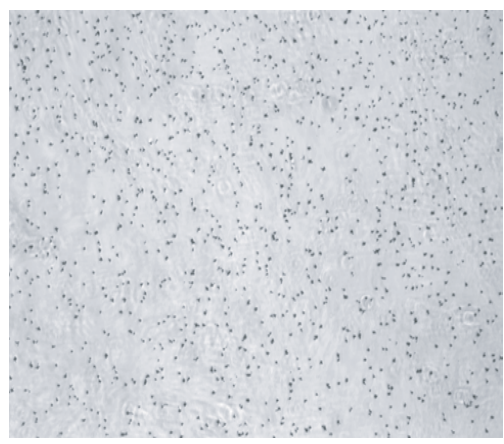

(b)

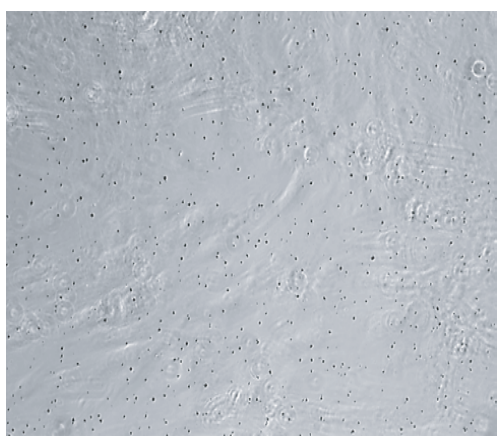

(e)

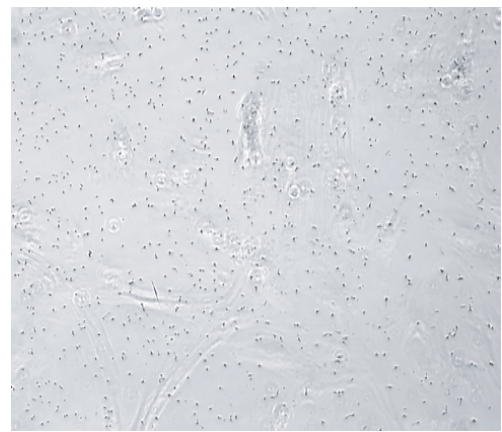

(c)

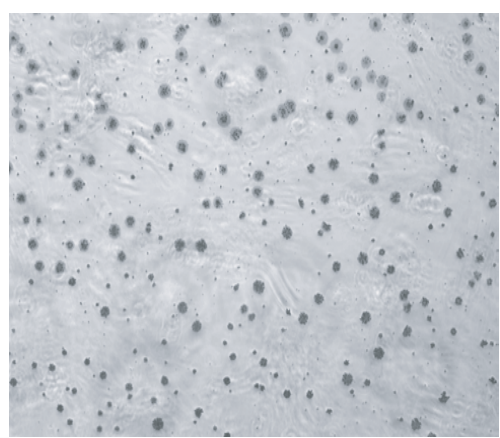

(f)

FIGURE 5. HMSC plated on COL I and vitronectin react positively by von Kossa staining. Cells were plated on tissue culture plastic (a), (b); COL I (c), (d); or VN (e), (f) in control medium (a), (c), and (e) or in OS supplemented medium (b), (d), and (f) for 16 days, then treated with silver nitrate solution. Presence of precipitated silver grains indicates the presence of calcium phosphate in the culture well (10× magnification).

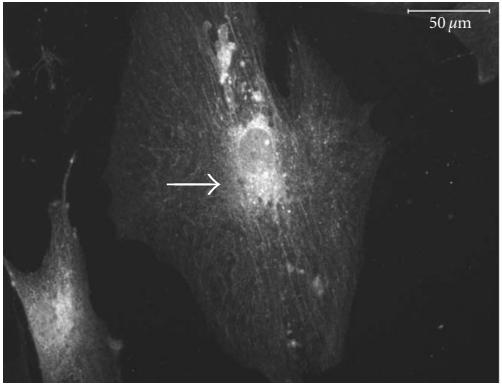

(a)

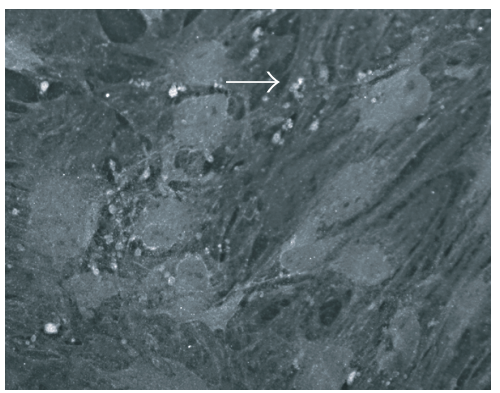

(b)

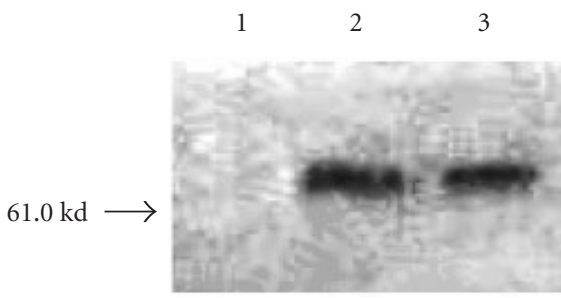

(c)

FIGURE 6. HMSC plated on COL I and VN express osteopontin. (a) and (b) Immunohistochemical staining of osteopontin in hMSC on day 16. Cells plated on COL I in DMEM (a) or on VN in DMEM (b) exhibit intracellular staining and deposits of osteopontin in the extracellular space. Arrows indicate concentrated aggregates of osteopontin. Bar in (a) $=50 \mu \mathrm{m}$, in (b) $=200 \mu \mathrm{m}$. (c) Western blot of cells cultured for eight days in control medium on tissue culture plastic (lane 1), COL I (lane 2), or VN (lane 3), and probed for osteopontin expression.

integrin receptors simultaneously and thereby activate a host of signaling pathways, which in turn leads to enhanced matrix mineralization. Thus, our data suggest a model wherein contact with COL I stimulates hMSC expression of osteogenic genes directly, while contact with
VN may stimulate expression of COL I; both $\alpha 1 \beta 1$ and $\alpha \mathrm{v} \beta 3$ integrins contribute to osteogenic differentiation via distinct mechanisms.

Exactly how ECM contact influences expression of osteogeneic genes is not known. However, considerable 


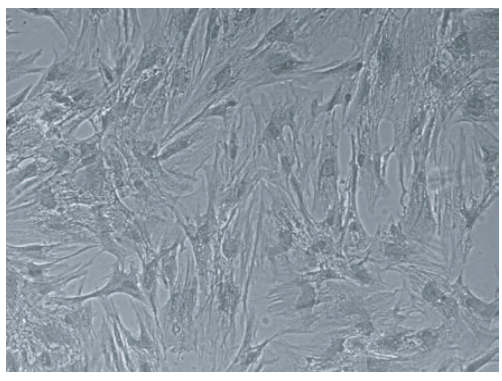

(a)

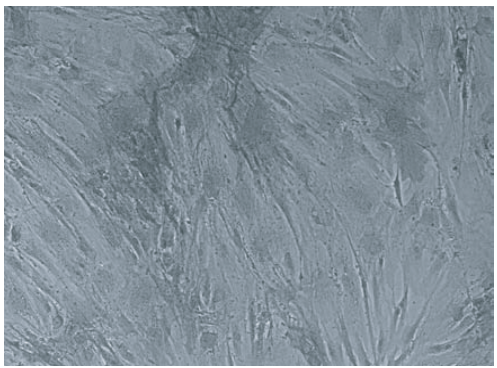

(c)

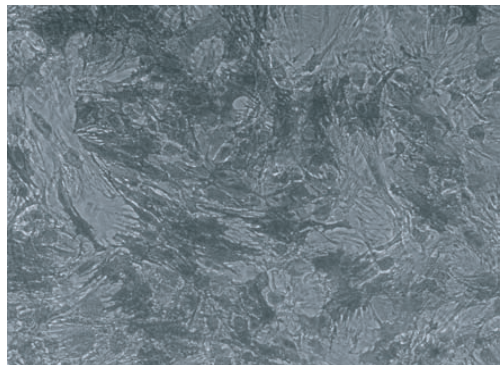

(b)

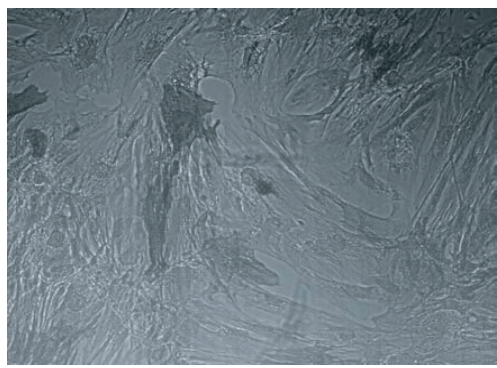

(d)

Figure 7. HMSC plated on COL I or VN express alkaline phosphatase activity. Cells were plated on tissue culture plastic (a), COL I (c), and vitronectin (d) in control media for 16 days then stained for alkaline phosphatase activity. Cultures were plated on tissue culture plastic in osteogenic supplement medium (b) as a positive control. Cells expressing alkaline phosphatase appear dark in phase contrast micrographs (10× magnification).

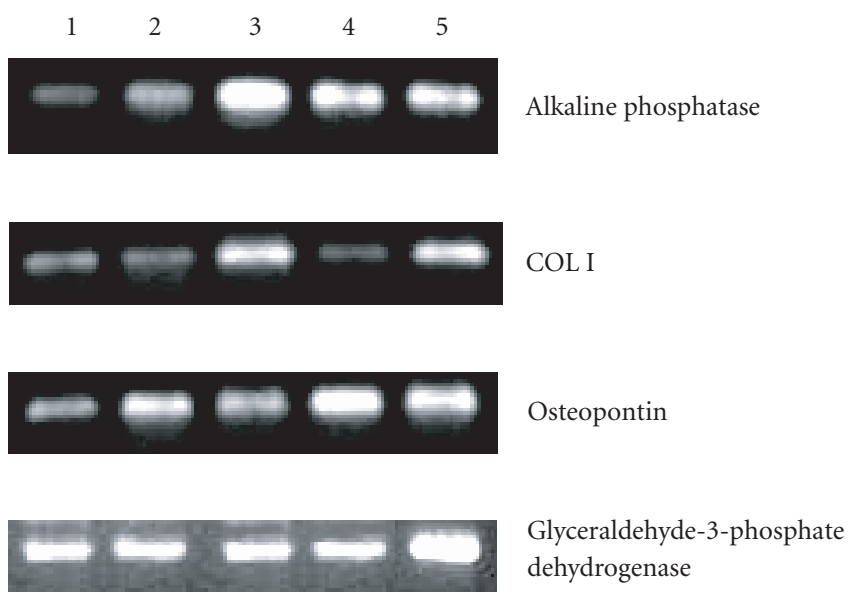

Figure 8. HMSC plated on COL I or VN express osteogenic genes. Indicated genes were amplified by RT-PCR from RNA isolated from hMSC plated for eight days in control medium on tissue culture plastic (lane 1), OS medium on tissue culture plastic (lane 2), control medium on COL I (lane 3), or control medium on VN (lane 4). As a positive control, RNA from eight-day cultures of fully differentiated osteoblasts was run in parallel (lane 5).

data implicates a role for the transcription factor Runx2/ CBFA-1 in controlling osteogenic gene expression [37, 38]. Activation of this transcription factor occurs via serine phosphorylation by extracellular regulated kinase
$1 / 2($ ERK 1/2) [39], which is a target of both growth factor receptors and integrin signaling pathways. Thus integrinECM contact may initiate a signaling cascade through ERK $1 / 2$ that leads to activation of Runx2/CBFA-1. The 
exact makeup of this signaling pathway has yet to be determined, but multiple studies have linked integrin binding to ERK $1 / 2$ activation in other cell types. The results of this study suggest that at least two distinct ECM proteins, and their corresponding integrin receptors, stimulate osteogenic differentiation without soluble OS factors, and strongly suggest that integrin signaling pathways directly control hMSC differentiation.

\section{REFERENCES}

[1] Jaiswal RK, Jaiswal N, Bruder SP, Mbalaviele G, Marshak DR, Pittenger MF. Adult human mesenchymal stem cell differentiation to the osteogenic or adipogenic lineage is regulated by mitogen-activated protein kinase. J Biol Chem. 2000;275(13):96459652.

[2] Pittenger MF, Mackay AM, Beck SC, et al. Multilineage potential of adult human mesenchymal stem cells. Science. 1999;284(5411):143-147.

[3] Jaiswal N, Haynesworth SE, Caplan AI, Bruder SP. Osteogenic differentiation of purified, cultureexpanded human mesenchymal stem cells in vitro. J Cell Biochem. 1997;64(2):295-312.

[4] Mackay AM, Beck SC, Murphy JM, Barry FP, Chichester CO, Pittenger MF. Chondrogenic differentiation of cultured human mesenchymal stem cells from marrow. Tissue Eng. 1998;4(4):415-428.

[5] Bruder SP, Jaiswal N, Haynesworth, SE. Growth kinetics, self-renewal, and the osteogenic potential of purified human mesenchymal stem cells during extensive subcultivation and following cryopreservation. J Cell Biochem. 1997;64(2):278-294.

[6] Aubin JE. Advances in the osteoblast lineage. Biochem Cell Biol. 1998;76(6):899-910.

[7] Doi M, Nagano A, Nakamura Y. Genome-wide screening by cDNA microarray of genes associated with matrix mineralization by human mesenchymal stem cells in vitro. Biochem Biophys Res Commun. 2002;290(1):381-390.

[8] Higuchi C, Myoui A, Hashimoto N, et al. Continuous inhibition of MAPK signaling promotes the early osteoblastic differentiation and mineralization of the extracellular matrix. J Bone Miner Res. 2002; 17(10):1785-1794.

[9] Cheng SL, Lai CF, Fausto A, et al. Regulation of alphaVbeta3 and alphaVbeta5 integrins by dexamethasone in normal human osteoblastic cells. J Cell Biochem. 2000;77(2):265-276.

[10] Ng PC, Lam CW, Wong GW, et al. Changes in markers of bone metabolism during dexamethasone treatment for chronic lung disease in preterm infants. Arch Dis Child Fetal Neonatal Ed. 2002;86(1): F49-F54.

[11] Aubin JE. Regulation of osteoblast formation and function. Rev Endocr Metab Disord. 2001;2(1):8194.
[12] Candeliere GA, Liu F, Aubin JE. Individual osteoblasts in the developing calvaria express different gene repertoires. Bone. 2001;28(4):351-361.

[13] Bortell R, Barone LM, Tassinari MS, Lian JB, Stein GS. Gene expression during endochondral bone development: evidence for coordinate expression of transforming growth factor beta 1 and collagen type I. J Cell Biochem. 1990;44(2):81-91.

[14] Pace JM, Chitayat D, Atkinson M, Wilcox WR, Schwarze U, Byers PH. A single amino acid substitution (D1441Y) in the carboxyl-terminal propeptide of the proalpha1(I) chain of type I collagen results in a lethal variant of osteogenesis imperfecta with features of dense bone diseases. J Med Genet. 2002;39(1):23-29.

[15] Aszodi A, Bateman JF, Gustafsson E, Boot-Handford R, Fassler R. Mammalian skeletogenesis and extracellular matrix: what can we learn from knockout mice? Cell Struct Funct. 2000;25(2):73-84.

[16] Mizuno M, Kuboki Y. Osteoblast-related gene expression of bone marrow cells during the osteoblastic differentiation induced by type I collagen. J Biochem (Tokyo). 2001;129(1):133-138.

[17] Xiao G, Wang D, Benson MD, Karsenty G, Franceschi RT. Role of the alpha2-integrin in osteoblast-specific gene expression and activation of the Osf2 transcription factor. J Biol Chem. 1998; 273(49):32988-32994.

[18] Schwartz MA, Ginsberg MH. Networks and crosstalk: integrin signalling spreads. Nat Cell Biol. 2002;4(4):E65-E68.

[19] Plopper GE, Domanico SZ, Cirulli V, Kiosses WB, Quaranta V. Migration of breast epithelial cells on Laminin-5: differential role of integrins in normal and transformed cell types. Breast Cancer Res Treat. 1998;51(1):57-69.

[20] Plopper GE, McNamee HP, Dike LE, Bojanowski $\mathrm{K}$, Ingber DE. Convergence of integrin and growth factor receptor signaling pathways within the focal adhesion complex. Mol Biol Cell. 1995;6(10):13491365.

[21] Bills CE, Eisenberg H, Pallante SL. Complexes of organic acids with calcium phosphate: the von Kossa stain as a clue to the composition of bone mineral. Johns Hopkins Med J. 1971;128(4):194-207.

[22] Kato Y, Boskey A, Spevak L, Dallas M, Hori M, Bonewald LF. Establishment of an osteoid preosteocyte-like cell MLO-A5 that spontaneously mineralizes in culture. J Bone Miner Res. 2001;16(9): 1622-1633.

[23] Frank O, Heim M, Jakob M, et al. Real-time quantitative RT-PCR analysis of human bone marrow stromal cells during osteogenic differentiation in vitro. $J$ Cell Biochem. 2002;85(4):737-746.

[24] Kessler G, Wolfman M. An automated procedure for the simultaneous determination of calcium and phosphorus. Clin Chem. 1964;10:686703. 
[25] Christenson RH. Biochemical markers of bone metabolism: an overview. Clin Biochem. 1997;30(8): 573-593.

[26] Globus RK, Moursi A, Zimmerman D, Lull J, Damsky C. Integrin-extracellular matrix interactions in connective tissue remodeling and osteoblast differentiation. ASGSB Bull. 1995;8(2):1928.

[27] Moursi AM, Globus RK, Damsky CH. Interactions between integrin receptors and fibronectin are required for calvarial osteoblast differentiation in vitro. J Cell Sci. 1997;110(pt 18):2187-2196.

[28] Moursi AM, Damsky CH, Lull J, et al. Fibronectin regulates calvarial osteoblast differentiation. J Cell Sci. 1996;109(pt 6):1369-1380.

[29] Yu YM, Becvar R, Yamada Y, Reddi AH. Changes in the gene expression of collagens, fibronectin, integrin and proteoglycans during matrix-induced bone morphogenesis. Biochem Biophys Res Commun. 1991;177(1):427-432.

[30] Conget PA, Minguell JJ. Phenotypical and functional properties of human bone marrow mesenchymal progenitor cells. J Cell Physiol. 1999;181(1):6773.

[31] Guo Z, Yang J, Liu X, et al. Biological features of mesenchymal stem cells from human bone marrow. Chin Med J (Engl). 2001;114(9):950-953.

[32] Majumdar MK, Thiede MA, Mosca JD, Moorman M, Gerson SL. Phenotypic and functional comparison of cultures of marrow-derived mesenchymal stem cells (MSCs) and stromal cells. J Cell Physiol. 1998;176(1):57-66.

[33] Gronthos S, Simmons PJ, Graves SE, Robey PG. Integrin-mediated interactions between human bone marrow stromal precursor cells and the extracellular matrix. Bone. 2001;28(2):174-181.

[34] Davis GE. Affinity of integrins for damaged extracellular matrix: alpha v beta 3 binds to denatured collagen type I through RGD sites. Biochem Biophys Res Commun. 1992;182(3):1025-1031.

[35] Shea LD, Wang D, Franceschi RT, Mooney DJ. Engineered bone development from a pre-osteoblast cell line on three-dimensional scaffolds. Tissue Eng. 2000;6(6):605-617.

[36] Caltabiano S, Hum WT, Attwell GJ, et al. The integrin specificity of human recombinant osteopontin. Biochem Pharmacol. 1999;58(10):15671578.

[37] Franceschi RT. The developmental control of osteoblast-specific gene expression: role of specific transcription factors and the extracellular matrix environment. Crit Rev Oral Biol Med. 1999;10(1):4057.

[38] Franceschi RT, Xiao G. Regulation of the osteoblastspecific transcription factor, Runx2: responsiveness to multiple signal transduction pathways. J Cell Biochem. 2003;88(3):446-454.
[39] Xiao G, Gopalakrishnan R, Jiang D, Reith E, Benson MD, Franceschi RT. Bone morphogenetic proteins, extracellular matrix, and mitogen-activated protein kinase signaling pathways are required for osteoblast-specific gene expression and differentiation in MC3T3-E1 cells. J Bone Miner Res. 2002; 17(1):101-110.

* Corresponding author.

E-mail: ploppg@rpi.edu

Fax: +1 518276 2162; Tel: +1 5182768288 

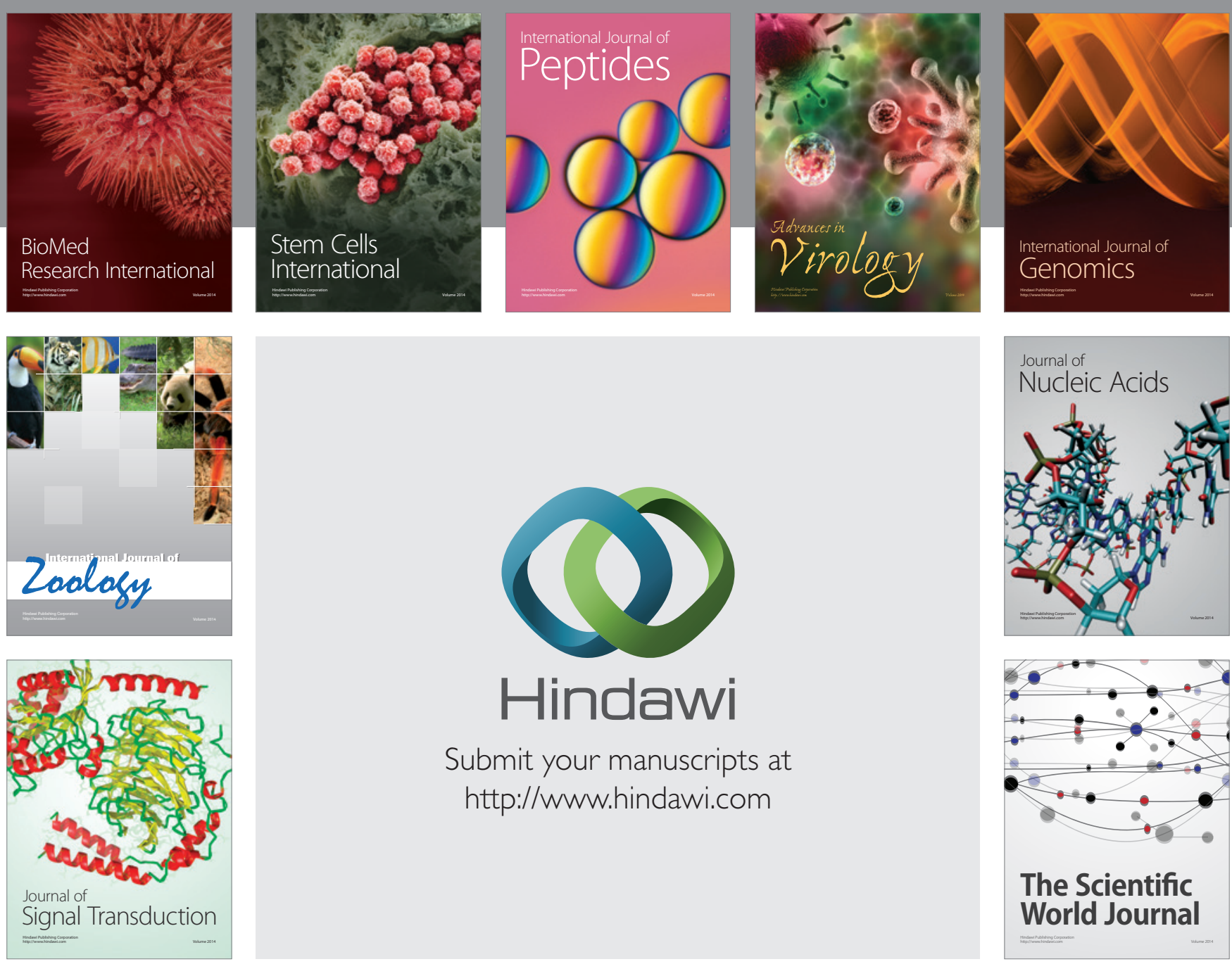

Submit your manuscripts at

http://www.hindawi.com
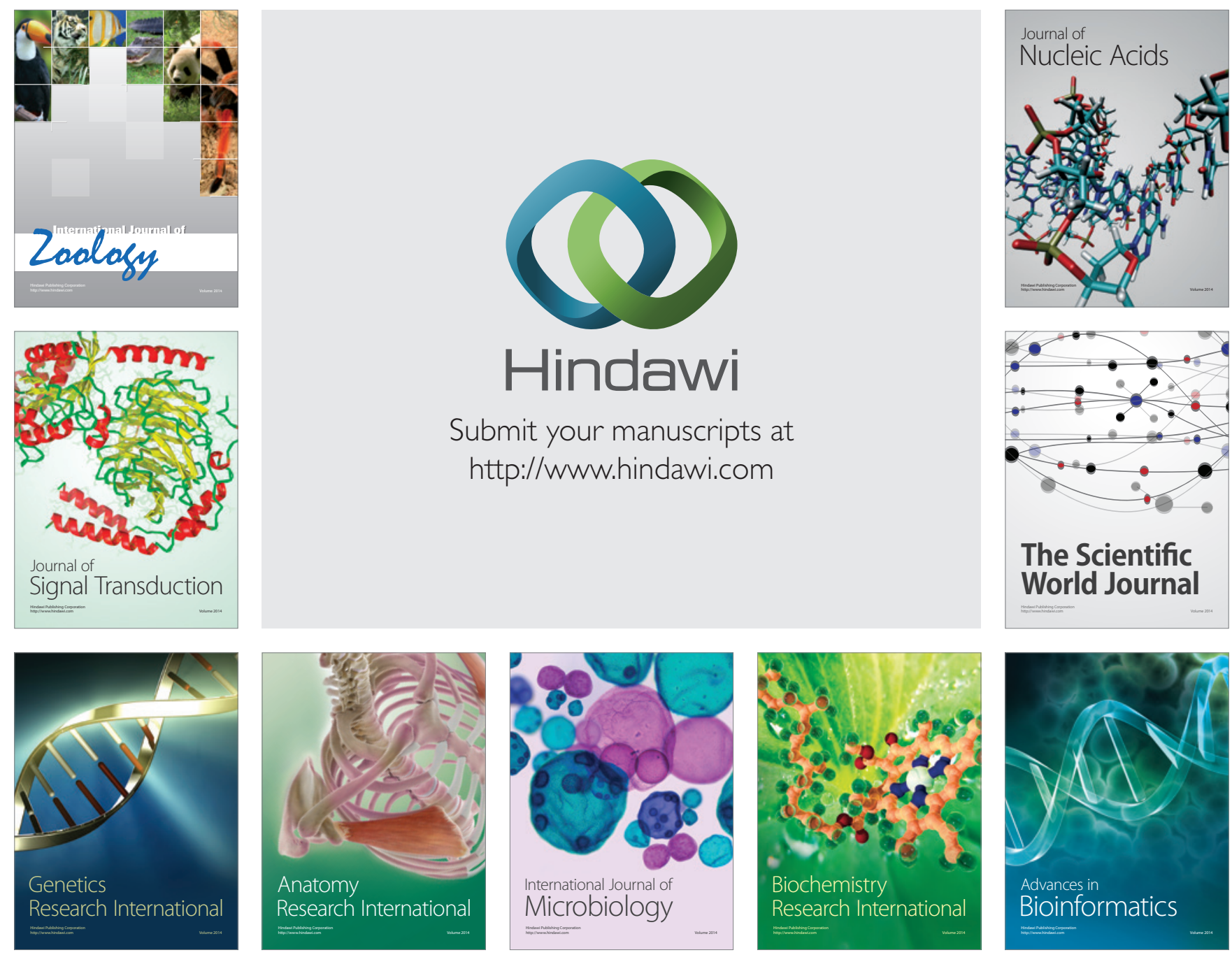

The Scientific World Journal
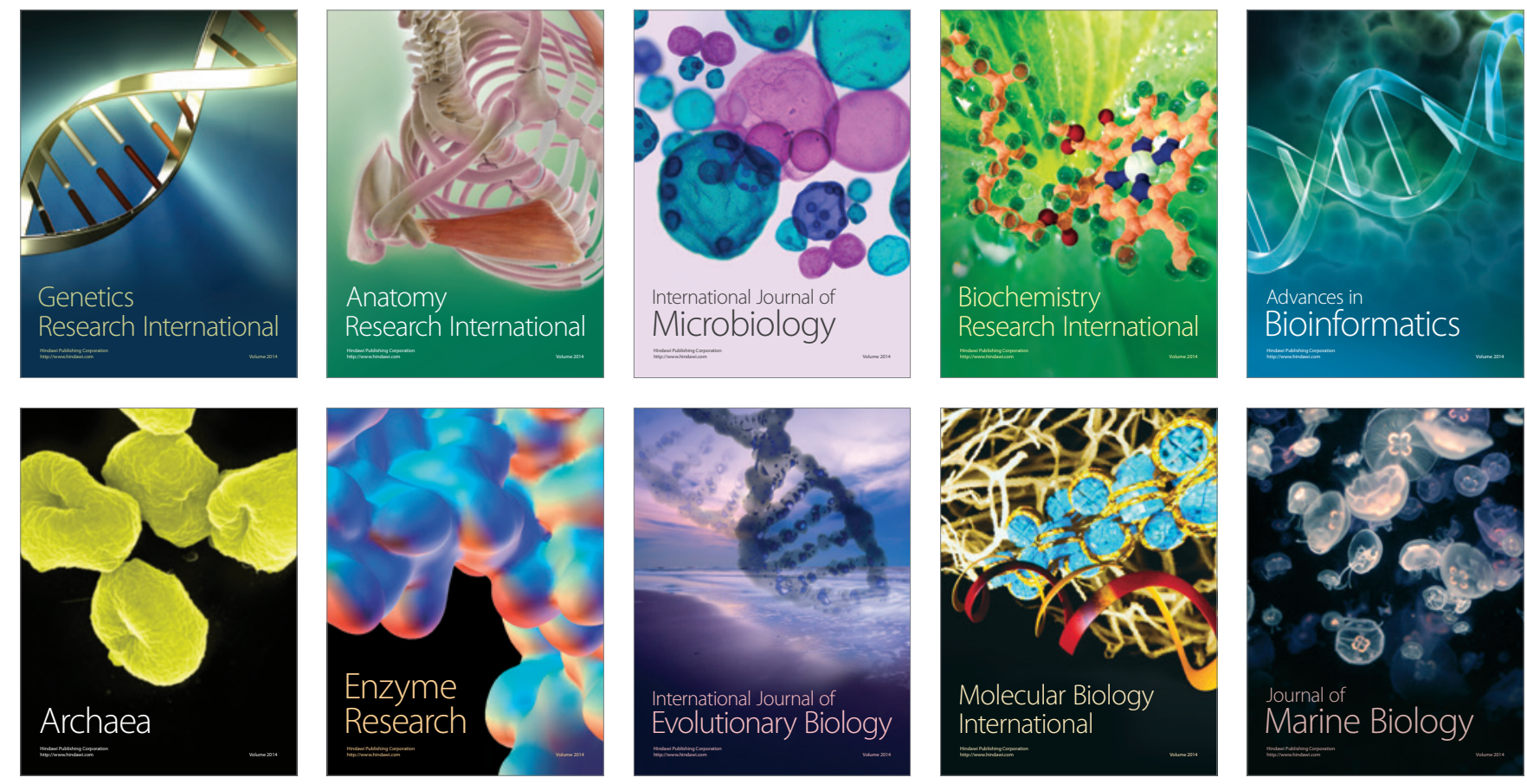